\title{
Superconformal Quantum Mechanics via Wigner-Heisenberg Algebra
}

\author{
H. L. Carrion ${ }^{\ddagger}$ and R. de Lima Rodrigues ${ }^{\alpha}$ \\ $\ddagger$, $\alpha$ Centro Brasileiro de Pesquisas Físicas \\ Rua Dr. Xavier Sigaud, 150 \\ CEP 22290-180, Rio de Janeiro-RJ, Brazil \\ - Instituto de Física, Universidade Federal do Rio de Janeiro \\ caixa postal 68528, 21945-970 Rio de Janeiro \\ RJ, Brazil. \\ ${ }^{\alpha}$ Departamento de Ciências Exatas e da Natureza \\ Universidade Federal da Paraíba, Cajazeiras - PB, 58.900-000, Brazil
}

\begin{abstract}
We show the natural relation between the Wigner Hamiltonian and the conformal Hamiltonian. It is presented a model in (super)conformal quantum mechanics with (super)conformal symmetry in the Wigner-Heisenberg algebra picture $\left[x, p_{x}\right]=i(1+c \mathbf{P})$ ( $\mathbf{P}$ being the parity operator). In this context, the energy spectrum, the Casimir operator, creation and annihilation operators are defined. This superconformal Hamiltonian is similar to the super-Hamiltonian of the Calogero model and it is also an extension of the super-Hamiltonian for the Dirac Oscillator.
\end{abstract}

E-mails: hleny@cbpf.br; rafael@df.ufcg.edu.br; 


\section{INTRODUCTION}

The problem of unifying quantum mechanics and gravity is one of the greatest unsolved problems in physics. In this context, the quantum mechanical black holes provide an arena in which quantum mechanics and gravity meet head on. The conformally-invariant quantum mechanical model was investigated firstly by Alfaro et al. [1] and an extended superconformal quantum mechanics has recently been discovered as a superconformal structure in multi black-hole quantum mechanics (see [2-5] and references therein). Also, some super-conformal models are sigma models that describe the propagation of a non-relativistic spinning particle in a curved background [6]. It was recently conjectured by Gibbons and Townsend that large $n$ limit of an $N=4$ superconformal extension of the $n$ particle Calogero model [7] might provide a description of the extreme Reissner-Nordström black hole near the horizon. In addition, the relation between the superconformal mechanics and the nonlinear supersymmetry we can see in [8].

In 1950, Wigner [9] proposed the interesting question, "Do the equations of motion determine the quantum-mechanical commutation relations?" and he found as an answer a generalized quantum rule for the one-dimensional harmonic oscillator. In the next year, Yang [10] found the coordinate representation for the linear momentum operator which realizes this aforementioned generalized quantum rule. Yang's wave mechanical description was further studied by Ohnuki et al. [11] and Mukunda et al. [12]. Jayaraman-Rodrigues have identified the free parameter of the Celka-Hussin's model with that of the Wigner parameter of a related super-realized general 3D Wigner oscillator system satisfying a generalized (super) quantum commutation relation of the $\sigma_{3}$-deformed Heisenberg algebra [13,14]. Recently, the deformed Wigner-Heisenberg (WH) oscillator algebra has been investigated in the context of the generalized statistics (introduced in physics in the form of parastatistics as an extension of the Bose and Fermi statistics) $[15,16]$. On the other hand, the elements of the conformal group can be represented in terms of ladder operators of deformed quantum oscillators [17] and the WH-algebra has also been investigated in connection with noncommutative geometry $[18,19]$.

In this paper, firstly we found the simple connection between the Wigner Hamiltonian and the conformal Hamiltonian of Ref. [2]. We proceed by showing the interesting new structures in conformal quantum mechanics in the WH picture. It is introduced the new well defined conformal Hamiltonian (22), its energy spectrum, the Casimir operator, raising (or creation) and the lowering (or annihilation) operators using the Wigner-Heisenberg algebra. It is shown, for example, that the eigenvalues of this conformal Hamiltonian is dependent of the Wigner parameter $c$ and the eigenvalues of the parity operator $P$. When $c=0$ we obtain the usual conformal Hamiltonian structure. Moreover, we present the new superconformal Hamiltonian with Wigner-Heisenberg algebra structure. In the same way, we study the energy spectrum and construct the supersymmetric Casimir operator. Our motivation is its potential application in (multi) black-hole quantum mechanics $[2,7]$ with the possibilities to introduce new structures in this subject.

This work is organized as follows. In Sec. II we start by summarizing the essential features of the formulation of $\mathbf{P}$-deformed Wigner-Heisenberg oscillator algebra. We discuss the connection between the usual conformal Hamiltonian and the Wigner Hamiltonian. In

Sec. III we present the conformal quantum mechanics based on the WH-algebra and discuss 
the eigenvector problem by defining the Casimir operator, the ladder and the annihilation operators. In Sec. IV we present the superconformal Hamiltonian based on the WH-algebra picture and define the supercharged operator in the Yang representation. We compute the superconformal algebra and discuss the eigenvalue and the eigenvector problems in the supersymmetric context. As in the bosonic case, in the supersymmetric case we defined the raising and the lowering operators and then we discuss the eigenvalue problem for the well defined superconformal Hamiltonian. In figures 1 and 2 the supersymmetric potentials for each specific parity operator eigenvalue are plotted.

\section{THE P-DEFORMED HEISENBERG ALGEBRA}

In order to make the paper self-contained we present a short discussion of the $P$-deformed Heisenberg algebra. The Wigner Hamiltonian expressed in the symmetrized bilinear form in terms of the mutually adjoint operators $\hat{a}^{ \pm}$, is defined by

$$
\hat{H}_{W}=\frac{1}{2}\left(\hat{p}_{x}^{2}+\hat{x}^{2}\right)=\frac{1}{2}\left[\hat{a}^{-}, \hat{a}^{+}\right]_{+}=\frac{1}{2}\left(\hat{a}^{-} \hat{a}^{+}+\hat{a}^{+} \hat{a}^{-}\right),
$$

where

$$
\hat{a}^{ \pm}=\frac{1}{\sqrt{2}}\left( \pm i \hat{p}_{x}-\hat{x}\right) .
$$

Wigner showed that the Heisenberg's equations of motion

$$
\left[\hat{H}_{W}, \hat{a}^{ \pm}\right]_{-}= \pm \dot{\hat{a}}^{ \pm}
$$

do not necessarily entail in the usual quantum rule

$$
\left[\hat{a}^{-}, \hat{a}^{+}\right]_{-}=1 \Rightarrow\left[\hat{x}, \hat{p}_{x}\right]_{-}=i, \quad \hbar=1,
$$

but a more general quantum rule [10-12] given by

$$
\left[\hat{a}^{-}, \hat{a}^{+}\right]_{-}=1+c \hat{R} \Longrightarrow\left[\hat{x}, \hat{p}_{x}\right]_{-}=i(1+c \hat{R}),
$$

where $c$ is a real constant, called Wigner parameter, related to the ground state energy $E_{W}^{(0)} \geq 0$ by virtue of the positive semi-definite form of $\hat{H}_{W}{ }^{1}$

$$
|c|=2 E_{W}^{(0)}-1
$$

The basic (anti-)commutation relations (1) and (3), together with the derived relation (5) constitute $\mathbf{P}$-deformed Wigner-Heisenberg algebra or in short the WH-algebra. The WH-algebra can also be obtained by the requirement that $\hat{x}$ satisfies the classical equation of motion $(\ddot{\hat{x}}+\hat{x}=0)$.

Note that $\hat{R}$ is an abstract operator satisfying the properties

\footnotetext{
${ }^{1}$ Note that the case $c=0$ corresponds to the usual oscillator with $E^{(0)}=\frac{1}{2}, \quad \hbar=\omega=1$.
} 


$$
\left[\hat{R}, \hat{a}^{ \pm}\right]_{+}=0 \Rightarrow\left[\hat{R}, \hat{H}_{W}\right]_{-}=0 ; \quad \hat{R}^{\dagger}=\hat{R}^{-1}=\hat{R}, \quad \hat{R}^{2}=1
$$

Also, we have

$$
\begin{aligned}
\hat{H}_{W} & =\hat{a}^{+} \hat{a}^{-}+\frac{1}{2}(1+c \hat{R}) \\
& =\hat{a}^{-} \hat{a}^{+}-\frac{1}{2}(1+c \hat{R}) .
\end{aligned}
$$

In the mechanical representation first investigated by Yang [10], $\hat{R}$ is realized by the parity operator $\mathbf{P}$ :

$$
\mathbf{P}|x>=|-x>\Rightarrow[\mathbf{P}, x]_{+}=0, \quad\left[\mathbf{P}, p_{x}\right]_{+}=0, \quad \mathbf{P}^{\dagger}=\mathbf{P}^{-1}=\mathbf{P}, \quad \mathbf{P}^{2}=1 .
$$

Indeed, Yang [10] found the coordinate representation for the momentum operator $p_{x}$ as given by

$$
\begin{gathered}
\hat{p}_{x} \longrightarrow p_{x}=p+i \frac{c}{2 x} \mathbf{P}, \quad \hat{x} \longrightarrow x, \quad p=-i \frac{d}{d x} \\
\hat{a}^{ \pm} \longrightarrow a_{\frac{c}{2}}^{ \pm}=\frac{1}{\sqrt{2}}\left( \pm \frac{d}{d x} \mp \frac{c}{2 x} \mathbf{P}-x\right) .
\end{gathered}
$$

Yang's wave mechanical description was further investigated in $[11,12]$.

The $\mathbf{P}$-deformed Heisenberg algebra is based on the following general quantum rule [10]

$$
\left[\hat{a}^{-}, \hat{a}^{+}\right]_{-}=1+c \mathbf{P} \Longrightarrow\left[\hat{x}, \hat{p}_{x}\right]_{-}=i(1+c \mathbf{P}),
$$

where

$$
\left[\mathbf{P}, \hat{a}^{ \pm}\right]_{+}=0 \Rightarrow\left[\mathbf{P}, \hat{H}_{W}\right]_{-}=0 .
$$

When we replace the equation (10) into the equation (1) for the Wigner Hamiltonian we obtain

$$
\hat{H}_{W-}=\frac{1}{2}\left\{p^{2}+x^{2}+\frac{1}{4 x^{2}}\left(c^{2}+2 c\right)\right\}
$$

where the parity operator has been taken the value -1 .

If we consider the following conformal Hamiltonian (equation (2.6) of ref. [2])

$$
L_{0}=\frac{p^{2}}{2}+\frac{g}{2 x^{2}}+\frac{x^{2}}{2}
$$

and choose $c^{2}+2 c=4 g$ in (14) then the Wigner Hamiltonian is equal the conformal Hamiltonian (15) in the coordinate representation

$$
L_{0}=\hat{H}_{W_{-}},
$$

for the case $\mathbf{P} \rightarrow-1$. This is a simple observation but leads to new interesting results. Note that introducing the more general quantum rule (5) we are providing conformal symmetry to the Wigner Hamiltonian. The energy spectrum of the Hamiltonian $L_{0}$ ( or $\hat{H}_{W-}$ ) is well defined. The eigenstates of $L_{0}$ form an infinite tower above the ground state, in integer steps $[1,2]$.

On the other hand, it is important to comment that there exists a hidden supersymmetry in the WH-algebra structure. If we define as the bosonic generators $\left\{\hat{H}_{W},\left(\hat{a}^{+}\right)^{2},\left(\hat{a}^{-}\right)^{2}\right\}$ and the fermionic ones as $\left\{\hat{a}^{+}, \hat{a}^{-}\right\}$then they close the $\operatorname{osp}(1 / 2)$ superalgebra. 


\section{CONFORMAL SYMMETRY IN THE WIGNER-HEISENBERG PICTURE}

In this section we study the Hamiltonian with conformal symmetry in the WH-algebra picture. This is a modified version of the usual conformal Hamiltonian with a standard canonical quantum rule $\left[\hat{x}, \hat{p}_{x}\right]=\imath[1,2]$. Let us define the new Hamiltonian

$$
H=\frac{p_{x}^{2}}{2}+\frac{g}{2 x^{2}}
$$

where $g$ is a coupling constant and $p_{x}$ is defined in (10) with new quantum rule (12). This Hamiltonian (17) commutes with the parity operator $\mathbf{P}$.

Next let us introduce the operators

$$
\begin{aligned}
& D=\frac{p_{x} x+x p_{x}}{2}, \\
& K=\frac{x^{2}}{2} .
\end{aligned}
$$

We can demonstrate that these three operators satisfy the following $S L(2, \mathbf{R})$ algebra

$$
[H, D]=-2 i H, \quad[H, K]=-i D, \quad[K, D]=2 i K
$$

where $D$ is known to be the generator of dilatation (it generates the re-scalings $x \rightarrow \gamma x$, $\left.p_{x} \rightarrow p_{x} / \gamma\right)$ and $K$ is the generator of special conformal transformations. Since $D$ and $K$ do not commute with the Hamiltonian $H$, they do not generate symmetries in the usual sense of relating the degenerate states. Rather they can be used to relate states with different eigenvalues of $H(17)$.

It is possible to show in any quantum mechanics with operators obeying the $S L(2, \mathbf{R})$ algebra (19), that if $|\chi\rangle$ is a state of energy $E$, then $e^{\imath \alpha D}$ is a state for the energy $e^{2 \alpha} E$. Thus, if there is a state of nonzero energy then the spectrum is continuous. Note that this result

provides the spectrum of Hamiltonian $H$ (17) to be continuous and then its eigenstates are not normalizable.

Let us consider the following linear combinations

$$
\begin{aligned}
L_{+} & =\frac{1}{2}(H-K+i D), \\
L_{-} & =\frac{1}{2}(H-K-i D), \\
L_{0} & =\frac{1}{2}(H+K) .
\end{aligned}
$$

The generators (20) satisfy the $S L(2, \mathbf{R})$ algebra (Virasoro form), with the following commutation relations

$$
\left[L_{-}, L_{+}\right]=2 L_{0}, \quad\left[L_{0}, L_{+}\right]=+L_{+} \quad\left[L_{0}, L_{-}\right]=-L_{-} .
$$

With the definition (18), (19) and (20), we have

$$
L_{0}=\frac{p_{x}^{2}}{4}+\frac{g}{4 x^{2}}+\frac{x^{2}}{4} .
$$


The potential energy for this operator (22) have an absolute minimum, then it has a discrete spectrum with normalized eigenstates. If $L_{0}$ satisfies the following eigenvalue equation

$$
L_{0}\left|n>=\epsilon_{n}\right| n>\text {, }
$$

and using the algebra (21) we see that $L_{-}$and $L_{+}$form the creation and annihilation operators for the Hamiltonian $L_{0}$, so that

$$
\epsilon_{n}=\epsilon_{0}+n, \quad n=0,1,2, \cdots .
$$

In other words, the eigenvalues of $L_{0}$ form an infinite tower above "the ground state", in integer steps.

The $S L(2, \mathbf{R})$ Casimir operator is given by

$$
L^{2}=L_{0}\left(L_{0}-1\right)-L_{+} L_{-},
$$

where

$$
\left[L^{2}, L_{+}\right]=\left[L^{2}, L_{-}\right]=\left[L^{2}, L_{0}\right]=0 .
$$

This Casimir operator in terms of the generators of the conformal group becomes

$$
L^{2}=\frac{H K+K H}{2}-\frac{D^{2}}{4} .
$$

Using the WH-algebra, the action of this Casimir operator on the eigenstates $|n\rangle$ gives us

$$
L^{2}=\frac{1}{16}[4 g-3-c(2 \mathbf{P}-c)]
$$

or

$$
L^{2}= \begin{cases}\frac{1}{16}\left[4 g-3-2 c+c^{2}\right], & \mathbf{P} \rightarrow+1, \\ \frac{1}{16}\left[4 g-3+2 c+c^{2}\right], & \mathbf{P} \rightarrow-1 .\end{cases}
$$

Defining $l_{0}$ by $L^{2}=l_{0}\left(l_{0}-1\right)$, from the relation (29) we obtain the "ground state" for this Casimir operator

$$
\ell_{0}= \begin{cases}\frac{1 \pm \sqrt{g+\frac{1}{4}(1-c)^{2}}}{2}, & \mathbf{P} \rightarrow 1 \\ \frac{1 \pm \sqrt{g+\frac{1}{4}(1+c)^{2}}}{2}, & \mathbf{P} \rightarrow-1\end{cases}
$$

and one can see that the coupling constant defined in (17) must satisfy $\left(g>-\frac{1}{4}(1 \pm c)^{2}\right)$.

When the constant $c$ vanishes the Casimir operator becomes $L^{2}=\frac{1}{16}(4 g-3)$, so that the ground state has the eigenvalue $\ell_{0}=\frac{1}{2}\left(1 \pm \sqrt{g+\frac{1}{4}}\right)$, which is a well known result for the usual conformal mechanics with the canonical commutation relation $[x, p]=i[2]$.

Note that $\epsilon_{0}=\ell_{0}$, or $\epsilon_{0}=1-\ell_{0}$ and

$$
\begin{aligned}
& L_{+}\left|n, \ell_{0}>=\sqrt{\left(\epsilon_{n}+\ell_{0}\right)\left(\epsilon_{n}-\ell_{0}+1\right)}\right| n+1, \ell_{0}>, \\
& L_{-}\left|n, \ell_{0}>=\sqrt{\left(\epsilon_{n}-\ell_{0}\right)\left(\epsilon_{n}+\ell_{0}-1\right)}\right| n-1, \ell_{0}>.
\end{aligned}
$$

From Eqs. (12) and (20), we can express $L_{+}$and $L_{-}$in terms of the operators $\hat{a}_{+}$and $\hat{a}_{-}$

$$
\begin{aligned}
& L_{+}=-\frac{1}{2} \hat{a}_{+}^{2}+\frac{g}{4 x^{2}}, \\
& L_{-}=-\frac{1}{2} \hat{a}_{-}^{2}+\frac{g}{4 x^{2}} .
\end{aligned}
$$




\section{THE SUPERCONFORMAL QUANTUM MECHANICS}

The superconformal quantum mechanic has been examined in [2]- [6]. Conformal sigma models may have applications in the context of $A d S / C F T$ correspondence with $A d S_{2} \times M$ background [20]. Another application for these models is in the study of the radial motion of test particle near the horizon of extremal Reissner-Nordström black holes [6,7]. Also, another interesting application of the superconformal symmetry is the treatment of the Dirac oscillator $[21,22]$.

In this section we introduce the explicit supersymmetry for the conformal Hamiltonian in the WH-algebra picture. Consider the supersymmetric generalization of $H$ (Eq. (17)) given by

$$
\mathcal{H}=\frac{1}{2}\left\{Q_{c}, Q_{c}^{\dagger}\right\}
$$

where the new supercharge operators are given in terms of the momentum Yang representation

$$
\begin{aligned}
& Q_{c}=\left(-i p_{x}+\frac{\sqrt{g}}{x}\right) \Psi^{\dagger}, \\
& Q_{c}^{\dagger}=\Psi\left(i p_{x}+\frac{\sqrt{g}}{x}\right),
\end{aligned}
$$

with $\Psi$ and $\Psi^{\dagger}$ being Grassmannian operators so that its anticommutator is $\left\{\Psi, \Psi^{\dagger}\right\}=$ $\Psi \Psi^{\dagger}+\Psi^{\dagger} \Psi=1$.

Explicitly the superconformal Hamiltonian becomes

$$
\mathcal{H}=\frac{1}{2}\left(\mathbf{1} p_{x}^{2}+\frac{\mathbf{1} g+\sqrt{g} B(1-c \mathbf{P})}{x^{2}}\right)
$$

where

$$
B=\left[\Psi^{\dagger}, \Psi\right]
$$

so that the parity operator is conserved, i.e., $[\mathcal{H}, \mathbf{P}]=0$.

When one introduces the following operators

$$
\begin{aligned}
S & =x \Psi^{\dagger}, \\
S^{\dagger} & =\Psi x,
\end{aligned}
$$

it can be shown that these operators together with the conformal quantum mechanics operators $D$ and $K$

$$
\begin{aligned}
D & =\frac{1}{2}\left(x p_{x}+p_{x} x\right), \\
K & =\frac{1}{2} x^{2},
\end{aligned}
$$


satisfy the deformed superalgebra $\operatorname{osp}(2 \mid 2)^{2}$, viz.,

$$
\begin{aligned}
& {[\mathcal{H}, D]=-2 \imath \mathcal{H}} \\
& {[\mathcal{H}, K]=-\imath D \text {, }} \\
& {[K, D]=2 \imath K,} \\
& \left\{Q_{c}, Q_{c}^{\dagger}\right\}=2 \mathcal{H} \text {, } \\
& \left\{Q_{c}, S^{\dagger}\right\}=-\imath D-\frac{1}{2} B(1+c \mathbf{P})+\sqrt{g}, \\
& \left\{Q_{c}^{\dagger}, S\right\}=+\imath D-\frac{1}{2} B(1+c \mathbf{P})+\sqrt{g}, \\
& {\left[Q_{c}^{\dagger}, D\right]=-\imath Q_{c}^{\dagger} \text {, }} \\
& {\left[Q_{c}^{\dagger}, K\right]=S^{\dagger} \text {, }} \\
& {\left[Q_{c}^{\dagger}, B\right]=2 Q_{c}^{\dagger} \text {, }} \\
& {\left[Q_{c}, K\right]=-S \text {, }} \\
& {\left[Q_{c}, B\right]=-2 Q_{c} \text {, }} \\
& {\left[Q_{c}, D\right]=-\imath Q_{c} \text {, }} \\
& {[\mathcal{H}, \mathcal{S}]=Q_{c}, \quad\left[\mathcal{H}, \mathcal{S}^{\dagger}\right]=-Q_{c}^{\dagger},} \\
& {\left[B, S^{\dagger}\right]=-2 S^{\dagger}, \quad[B, S]=2 S \text {, }} \\
& {[D, S]=-\imath S, \quad\left[D, S^{\dagger}\right]=-\imath S^{\dagger},} \\
& \left\{S^{\dagger}, S\right\}=2 K \text {, }
\end{aligned}
$$

where, $\mathcal{H}, \mathcal{D}, \mathcal{K}, \mathcal{B}$ are bosonic operators and $Q_{c}, Q_{c}^{\dagger}, S, S^{\dagger}$ are fermionic operators. This superalgebra was introduced by Plyushchay in the bosonization of supersymmetry context [23]. The supersymmetric extension of the conformal Hamiltonian $L_{0}$ (presented in the previous section) is

$$
\mathcal{H}_{0}=\frac{1}{2}(\mathcal{H}+K), \quad\left[\mathcal{H}_{0}, \mathbf{P}\right]=0 .
$$

The super-Hamiltonian $\mathcal{H}_{0}$ is similar to the supersymmetric Calogero interaction Hamiltonian associated with two-particle interaction. The possibility that the $n$-particle Calogero model ${ }^{3}$ might be relevant for a microscopic description of the extreme Reissner-Nordstrom black hole, has been discussed in Refs. [7,25]. Specifically, it was conjectured by Gibbons and Townsend that the large $n$ limit of $N=4$ superconformal version of the $n$-particle Calogero model might provide microscopical description of the Reissner-Nordstrom black hole near to the horizon [7]. Continuing with our discussion, now we are able to build up the ladder operators for the spectral resolution of the super-Hamiltonian $\mathcal{H}_{0}$. Note that defining

\footnotetext{
${ }^{2}$ Actually, this superalgebra is $\operatorname{osp}(2 \mid 2)$ when we fix $\mathbf{P}=1$ or $\mathbf{P}=-1$.

${ }^{3}$ Calogero model [24] describes the system of $n$ bosonic particles interacting through the inverse square and harmonic potential, it is completely integrable (at classical and quantum levels).
} 


$$
\begin{gathered}
M=Q_{c}-S, \\
Q=Q_{c}+S, \\
h \equiv \frac{1}{2}\left\{M, M^{\dagger}\right\}=2 \mathcal{H}_{0}+\frac{1}{2}(1+c \mathbf{P}) B-\sqrt{g}, \\
\tilde{h} \equiv \frac{1}{2}\left\{Q, Q^{\dagger}\right\}=2 \mathcal{H}_{0}-\frac{1}{2}(1+c \mathbf{P}) B+\sqrt{g},
\end{gathered}
$$

we obtain

$$
\begin{aligned}
{\left[h, Q^{\dagger}\right] } & =-2 Q^{\dagger}, \\
{[h, Q] } & =2 Q
\end{aligned}
$$

and

$$
\begin{aligned}
{\left[\tilde{h}, M^{\dagger}\right] } & =2 M^{\dagger} \\
{[\tilde{h}, M] } & =-2 M .
\end{aligned}
$$

Thus we see that $Q$ and $Q^{\dagger}, M$ and $M^{\dagger}$ are ladder operators associated to the SUSY Hamiltonian operators $h$ and $\tilde{h}$, respectively. We remark that since $h, \tilde{h}$ and $\mathcal{H}_{0}$ commute with each other, they form a set of mutually commuting operators. The superhamiltonian $h$ and $\tilde{h}$ are extensions of the ones in references $[21,22]$ for the superconformal Dirac oscillator.

The supersymmetric generalizations of the ladder operators defined in (20) in the bosonic case, are given by

$$
\begin{aligned}
\mathcal{L}_{-} \equiv-\frac{1}{4}\left\{M, Q^{\dagger}\right\} & =-\frac{1}{2}(H-K-\imath D), \\
\mathcal{L}_{+} \equiv-\frac{1}{4}\left\{M^{\dagger}, Q\right\} & =-\frac{1}{2}(H-K+\imath D)
\end{aligned}
$$

with the following commutation relations of the $s o(2,1)$ algebra

$$
\begin{aligned}
& {\left[\mathcal{L}_{+}, \mathcal{H}_{0}\right]=-\mathcal{L}_{+},} \\
& {\left[\mathcal{L}_{-}, \mathcal{H}_{0}\right]=+\mathcal{L}_{-},} \\
& {\left[\mathcal{L}_{+}, \mathcal{L}_{-}\right]=-2 \mathcal{H}_{0} .}
\end{aligned}
$$

The supersymmetric Casimir operator is given by

$$
\mathcal{L}^{2}=\mathcal{H}_{0}\left(\mathcal{H}_{0}-1\right)-\mathcal{L}_{+} \mathcal{L}_{-}
$$

Thus, the energy spectrum becomes

$$
\begin{aligned}
\mathcal{H}_{0} \mid m, s> & =\zeta_{m} \mid m, s> \\
\mathcal{L}^{2} \mid m, s> & =s(s-1) \mid m, s>
\end{aligned}
$$

with $\zeta_{m}=\zeta_{0}+m$. 
Since the eigenket $\mid m, s>$ is normalized, from (49) and (50) we obtain $s=s_{0}=\zeta_{0}$. In this case, we may write the analogous representations to the ladder operators given by Eq. $(31)$

$$
\begin{aligned}
& \mathcal{L}_{+}\left|m, s>=\alpha_{m+1}\right| m+1, s> \\
& \mathcal{L}_{-}\left|m, s>=\beta_{m-1}\right| m-1, s>
\end{aligned}
$$

where $\alpha_{m+1}$, and $\beta_{m-1}$ have a similar form to the bosonic case, given in the equations (31). Explicitly

$$
\mathcal{L}^{2}=\frac{1}{16}\left[(4 g-3-c(2 \mathbf{P}-c)) \mathbf{l}_{2 \times 2}+4 \sqrt{g} B(1-c \mathbf{P})\right]
$$

where

$$
\mathbf{l}_{2 \times 2}=\left[\begin{array}{ll}
1 & 0 \\
0 & 1
\end{array}\right], \quad B=\left[\begin{array}{cc}
-1 & 0 \\
0 & 1
\end{array}\right]=-\sigma_{3},
$$

and the parity operator must be substituted by its eigenvalues on the eigenstates $\mid m, s>$. In the case $\mathbf{P} \rightarrow+1$, on gets

$$
\mathcal{L}^{2}=\frac{1}{16}\left[\begin{array}{cc}
4 g-3-2 c+c^{2}-4 \sqrt{g}(1-c) & 0 \\
0 & 4 g-3-2 c+c^{2}+4 \sqrt{g}(1-c)
\end{array}\right]
$$

with the following eigenvalues

$$
\begin{aligned}
s(s-1) & =\frac{1}{16}\left(4 g-3-2 c+c^{2}-4 \sqrt{g}(1-c)\right) \\
s^{\prime}\left(s^{\prime}-1\right) & =\frac{1}{16}\left(4 g-3-2 c+c^{2}+4 \sqrt{g}(1-c)\right) .
\end{aligned}
$$

From equation (41) and (53) we obtain the SUSY conformal Hamiltonian in the coordinate representation

$$
\mathcal{H}_{01}=\frac{1}{4}\left[\begin{array}{cc}
p^{2}+x^{2}+\frac{c^{2}+2 c+4 g-4 \sqrt{g}(1-c)}{4 x^{2}} & 0 \\
0 & p^{2}+x^{2}+\frac{c^{2}+2 c+4 g+4 g \sqrt{g}(1-c)}{4 x^{2}}
\end{array}\right],
$$

with the following superconformal potential

$$
V_{01}(x) \equiv\left[\begin{array}{cc}
V_{01}(+) & 0 \\
0 & V_{01}(-)
\end{array}\right]=\frac{1}{4}\left(\left(x^{2}+\frac{c^{2}+2 c+4 g}{4 x^{2}}\right) \mathbf{1}_{2 \times 2}-\sigma_{3} \frac{\sqrt{g}(1-c)}{x^{2}}\right) .
$$

In Fig. 1 we plot $V_{01}(+)$, the bosonic part of this supersymmetric potential.

In the case $\mathbf{P} \rightarrow-1$ we get

$$
\mathcal{L}^{2}=\frac{1}{16}\left[\begin{array}{cc}
4 g-3+2 c+c^{2}-4 \sqrt{g}(1+c) & 0 \\
0 & 4 g-3+2 c+c^{2}+4 \sqrt{g}(1+c)
\end{array}\right] .
$$

Also, in this case 


$$
\mathcal{H}_{02}=\frac{1}{4}\left[\begin{array}{cc}
p^{2}+x^{2}+\frac{c^{2}-2 c+4 g-4 \sqrt{g}(1+c)}{4 x^{2}} & 0 \\
0 & p^{2}+x^{2}+\frac{c^{2}-2 c+4 g+4 \sqrt{g}(1+c)}{4 x^{2}}
\end{array}\right]
$$

with superconformal potential

$$
V_{02}(x)=\frac{1}{4}\left(\left(x^{2}+\frac{c^{2}-2 c+4 g}{4 x^{2}}\right) \mathbf{1}_{2 \mathrm{X} 2}-\sigma_{3} \frac{\sqrt{g}(1+c)}{x^{2}}\right) .
$$

In Fig. 2 we plot $V_{02}(+)$, the bosonic part of this supersymmetric potential.

Observe that we are using the convention that the operator number $N_{f}$ has the fermion number $n_{f}=0$ and is associated to the bosonic state and this eigenstate is given by

$$
\left(\begin{array}{l}
1 \\
0
\end{array}\right)
$$

Similarly, the eigenstates of $N_{f}$ with the fermion number $n_{f}=1$ is associated to the fermionic state, and is given by

$$
\left(\begin{array}{l}
0 \\
1
\end{array}\right)
$$

Finally, observing the supersymmetric Hamiltonians $\mathcal{H}_{01}$ and $\mathcal{H}_{02}$ we recall that the parameter $c$ is real and $g$ must be positive. With appropriate choice of these parameters we can recover various cases, for example the oscillator type superhamiltonian (without the presence of the proportional term $1 / x^{2}$ ) although the bosonic part without supersymmetry has this term $(g \neq 0)$. In this case the supersymmetric potential will not have a singularity at the origin of coordinates.

\section{CONCLUSION}

In this work, we have analyzed the connection between the conformal quantum mechanics $[1,2]$ and the Wigner-Heisenberg algebra $[10,11,13]$. With an appropriate relationship between the coupling constant $g$ and real constant $c$ one can identify the Wigner Hamiltonian with the conformal Hamiltonian $L_{0}$. The important result is that the introduction of the Wigner-Heisenberg algebra in the conformal quantum mechanics is still consistent with the conformal symmetry. The spectrum for the Casimir operator, the Hamiltonian $L_{0}$ and the ladder operators depend on the parity operator. We also investigated the supersymmetrization of this model, in that case we obtain a new spectra for the supersymmetric and conformal Hamiltonian of the Calogero interaction's type. In this case the spectrum for the super-Casimir operator and the superhamiltonian depend also on the parity operator. One motivation for this work is the future applications in the problems related to black holes $[2,7]$ and the construction of a supersymmetric quantum mechanics with conformal symmetry for the n-particles in the Wigner-Heisenberg picture. Let us point out that one can consider the same analysis implemented in this work for the Dirac oscillator, getting a generalization of the works $[21,26,27]$. 
Finally, it would be interesting to investigate the possible connection between the hidden supersymmetry in the $\mathrm{WH}$-algebra structure and the explicit supersymmetry implemented in the section 4, work in this direction is under current research.

\section{ACKNOWLEDGMENTS}

The authors are grateful to Prof. J. A. Helayel Neto for the kind hospitality at CBPFMCT. The authors are also partially supported by CNPq-CLAF. We are also grateful to

Harold Blas and F. Toppan for interesting discussions and suggestions. 


\section{REFERENCES}

[1] V. de Alfaro, S. Fubini and G. Furlan, Nuovo Cimento 34A (1976), 569.

[2] R. Britto-Pacumio, J. Michelson, A. Strominger and A. Volovich, Phys. Rev. D50 (2000), 43, het-th/9911066; hep-th/9908044.

[3] E. Deodato, G. Furlan and E. Gozzi, J. Math. Phys. 41 (2000), 8083, hep-th/9910220.

[4] V. P. Akulov and I. A. Pashnev, Theor. Math. Phys. 56 (1983), 862.

[5] S. Fubini and E. Rabinovici, Nucl. Phys. B 245 (1984), 17.

[6] P. Claus, M. Derix, R. kallosh, J. Kumar, P. Townsend and A. Van Proeyen, Phys. Rev. Lett. 81 (1998), 4553, hep-th/9804177.

[7] G. W. Gibbons and P. K. Townsend, Phys. Lett. B454 (1999), 187.

[8] C. Leiva and M. S. Plyushchay, JHEP 69 (2003), 0310.

[9] E. P. Wigner, Phys. Rev. 77 (1950), 711.

[10] L. M. Yang, Phys. Rev. 84 (1951), 788.

[11] Y. Ohnuki and S. Kamefuchi, J. Math. Phys. 19 (1978), 67; Y. Ohnuki and S. Watanabe, J. Math. Phys. 33 (1992), 3653.

[12] N. Mukunda, E. C. G. Sudarshan, J. K. Sharma and C. L. Mehta, J. Math. Phys. 21 (1980), 2386.

[13] J. Jayaraman and R. de Lima Rodrigues, J. Phys. A: Math. Gen. 23 (1990), 3123.

[14] J. Jayaraman and R. de Lima Rodrigues, Mod. Phys. Lett. A9 (1994), 1047.

[15] M. S. Plyushchay, Nucl. Phys. B491 (1997), 619; M. S. Plyushchay, Int. J. of Mod. Phys. A15 (2000), 3679.

[16] M. S. Plyushchay, Deformed Heisenberg algebra with reflection, anyons and supersimmetry of parabosons, hep-th/0006238.

[17] E. L. Da Graça, H. L. Carrion and R. de Lima Rodrigues, Braz. J. Phys. 33 (2003), 333, hep-th/0205167.

[18] R. C. King, T. D. Palev and N. I. Stoilova and J. Van der Jeugt, J. Phys. A: Math. Gen. 36 (2003), 4337, hep-th/0304136.

[19] R. C. King, T. D. Palev and N. I. Stoilova and J. Van der Jeugt, J. Phys. A: Math. Gen. A36 (2003), 11999.

[20] S. Bellucci, A. Galajinsky, E. Ivanov and S. Krivonos, Phys. Lett. B555 (2003), 99, hep-th/0212204.

[21] R. P. Martínez y Romero, Matías Moreno and A. Zentella, Phys. Rev. D43 (1991), 2036.

[22] R. P. Martinez y Romero and A. L. Salas Brito, J. Math. Phys. 33 (1992), 1831.

[23] M. S. Plyushchay, Mod. Phys. Lett. A11 (1996), 397.

[24] F. Calogero J. Math. Phys. 10 (1969), 2197.

[25] A. V. Galajinsky, Comments on $N=4$ Superconformal Extension of the Calogero Model, hep-th/0302156.

[26] M. Moshinsky and A. Szczepaniac, J. Phys. A: Math. Gen. 22 (1989), L817.

[27] R. de Lima Rodrigues and A. N. Vaidya, Dirac oscillator via R-deformed Heisenberg algebra, Proceedings of the XXIII Brazilian National Meeting on Particles and Fields (October/2002), site www.sbfisica.org.br/eventos/enfpc/xxiii/procs/trb0013.pdf, preprintCBPF-NF-030/02, hep-th/0301199. 


\section{FIGURES}

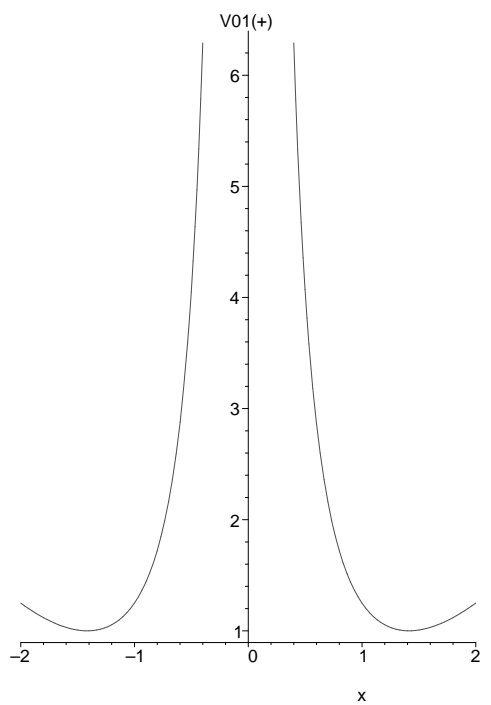

Fig 1. The bosonic part of the supersymmetric potential $V_{01}$, for $P=+1, c=2, g=1$.

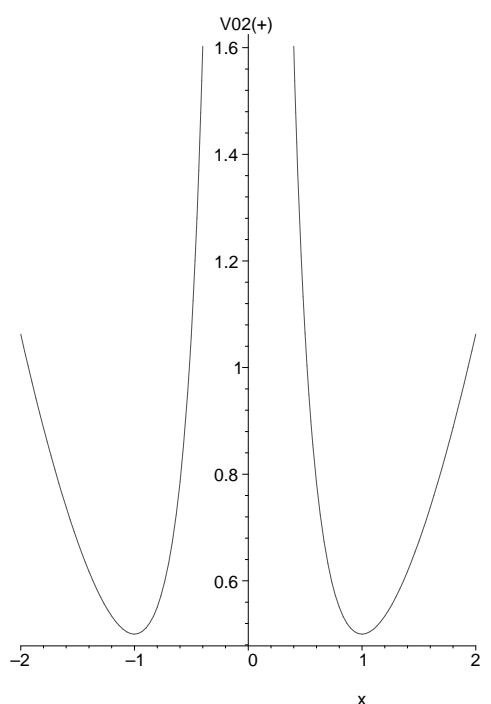

Fig 2. The bosonic part of the supersymmetric potential $V_{02}$, for $P=-1, c=2, g=1$. 